Originalveröffentlichung in: Katharina Münchberg, Christian Reidenbach (Hg.), Freundschaft. Theorien und Poetiken, München 2012, S. 97-116

\author{
JAN ASSMANN
}

\title{
Freundschaft, Feindschaft und Gemeinschaft im Alten Ägypten
}

\author{
„Der Untätige hat kein Gestern, \\ der für die Wahrheit Taube hat keinen Freund, \\ der Habgierige hat keinen Festtag."
}

„Es gibt keinen Freund für den, der für die Ma'at taub ist" “, liest man in einem altägyptischen Text aus dem 19. oder 18. vorchristlichen Jahrhundert. Es handelt sich um eine der seltenen altägyptischen Aussagen über Freundschaft, und sie ist - wie es für die altägyptische Dichtung kennzeichnend ist - so orakelhaft kurz gefasst, dass wir kaum etwas damit anfangen können. Dieser Satz erinnert in seiner Kompaktheit an jene japanischen Papierblumen, die Proust mit Erinnerungsspuren vergleicht: Man muss sie in Wasser legen und warten, bis sie sich darin auseinanderfalten, um erkennen zu können, um was es sich handelt. Mein Beitrag ist so ein Wasserbad, in das ich den ägyptischen Satz legen möchte, um ihn sich darin ausbreiten und von allen Seiten betrachten zu lassen.

\section{I.}

Zunächst müssen wir wissen, was „Ma'at“ bedeutet. ${ }^{2}$ Im Deutschen pflegen wir diesen Begriff mit „Wahrheit - Gerechtigkeit - Ordnung“ zu umschreiben. Ma'at ist das Richtige, das sich im Sprachlichen als Wahrheit, im Tun als Gerechtigkeit und in der Welt der Dinge als Ordnung manifestiert. Es handelt sich um den richtigen Zusammenhang der Menschen und der Dinge - darin sind für die Ägypter Wahrheit, Gerechtigkeit und Ordnung erfasst. Im Hinblick auf die Freundschaft ist Ma'at eine Sache der Sprache; anders könnte man nicht „taub“ für sie sein. Um für die Ma'at zugänglich zu sein, muss man hören, zuhören, verstehen, gehorchen können. Das ist offenbar als Gegensatz zu „,taub sein“ gemeint. Die „Wahrheit - Gerechtigkeit - Ordnung“ wird sprachlich kommuniziert, und wer wegen Taubheit von dieser Kommunikation ausgeschlossen ist, hat keinen Freund. Diese Engführung von Sprache und Freundschaft lässt an Aristoteles' berühmte Bestimmung des Menschen denken als das Tier, das Sprache hat (zoon logon echon) und das Tier, das in Gemeinschaft lebt (zoon politikon). Um in Gemeinschaft leben zu können, braucht der Mensch die Sprache, und er braucht sie nicht nur als ein sprechendes, sondern auch als ein hörendes, das heißt: ver-

Papyri Berlin $3023+3025$ und Ramesseum A = Papyrus 10499, s. Jan Assmann, Ma'at. Gerechtigkeit und Unsterblicbleeit im Alten Ägypten, München 1990, S. 60.

2 S. hierzu umfassend ebd. 
stehendes Wesen, denn worum es bei diesem Sprechen und Hören geht, sind die Grundlagen des Zusammenlebens, über die es sich zu verständigen gilt. Diese Grundlagen sind in dem ägyptischen Begriff der Ma'at zusammengefasst.

Zum Aufeinander-Hören gehört die Nähe, das Zusammenleben. In einer Welt ohne Post, Telefon und Internet ist sprachliche Kommunikation und damit Freundschaft ohne räumliche Nähe nicht denkbar. „Nichts“, schreibt Aristoteles,

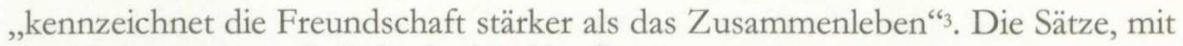
denen der Berliner Politologe Ekkehard Krippendorff diese Feststellung kommentiert, scheinen mir auch, und eigentlich noch genauer, auf die ägyptische Sentenz „Wer für die Ma'at taub ist, hat keinen Freund“ zu passen: „Dieses Zusammenleben“, schreibt Krippendorff, „schafft die Möglichkeit der Sprache, des Wortes. Wo dieses fehlt oder durch dauerhafte Entfernung schwindet, dann schwindet auch der Raum des Öffentlichen und mit ihm die Freundschaft. Und umgekehrt: wo die Freundschaft als ständig zu reproduzierende Kommunikation schwindet, da schwindet auch das Politische. Aristoteles sagt: ,Oft schon hat fehlender Austausch des Wortes die Freundschaft vernichtet'." "Nichts könnte genauer der ägyptischen Einschätzung der Freundschaft entsprechen als dieser Satz. Bedeutsam aber scheint mir, dass der ägyptische Satz die Beziehung zwischen Freundschaft und Sprache nicht am Sprechen, sondern am Zuhören festmacht. Offen zu sein für die Worte der Ma'at, das ist es, was den Freund ausmacht.

So beteuert auch der Tote, wenn er sich, wie die Ägypter glaubten, vor dem Totengericht zu verantworten hat: „[]ch war nicht taub gegen Worte der Wahr-

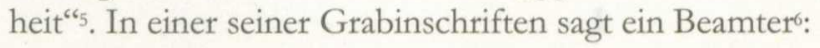

Ich freute mich über Worte der Ma'at, mein Abscheu war es, Lügen anzuhören.

Ich tat die Ma'at auf Erden soviele Male wie Haare auf dem Kopf sind.

Ich bin ein Gerechtfertigter an allen meinen Plätzen am Tag des (Toten-)Gerichts.

Worte der Ma'at sind Aussagen, die den richtigen Zusammenhang sprachlich darund herzustellen vermögen.

Zuhören, verstehen und gehorchen können ist für die Ägypter die oberste Tugend, die den Einzelnen nicht nur zum Freund, sondern überhaupt erst zum Menschen macht. Die Lehre des Ptahhotep, die wichtigste und klassische Ethik der alten Ägypter, schließt mit einer langen Diatribe über das Hören, aus der ich die wichtigsten Abschnitte zitieren möchte?:

\footnotetext{
Aristoteles, Nikomachische Ethik, übers. u. komm. von Franz Dirlmeier, Berlin 1979, 1158 .

4 Ekkehard Krippendorf, „Freundschaft als politische Kategorie“, in: ders., Die Kultur des Politischen. Wege aus den Diskeursen der Macht, Berlin 2009, S. 45-54; hier: S. 45-46.

5 Das Agyptische Totenbuch, übers. u. komm. von Gregoire Kolpaktchy, Frankfurt a. M. 1970, S. 125.

6 Inschrift des Anhurmose in seinem Grab in This (13. Jh. von Chr.), nach Assmann (1990), S. 105.

7 „Lehre des Ptahhotep“, in: Ma'at Konfurius Goethe. Drei Lebren für das richtige Leben, hrsg. v. Jan Assmann, Ekkehard Krippendorff, Helwig Schmidt-Glintzer, Frankfurt a. M. 2006, S. 46-48.
} 
Wenn du auf das hörst, was ich dir gesagt habe, dann werden alle deine Pläne an die Vorfahren heranreichen.

In der Art ihrer Ma'at liegt ihr Reichtum, und die Erinnerung an sie läuft noch um im Munde der Menschen, weil ihre Sprüche so vollkommen sind.

Jedes Wort wird überliefert, keines geht verloren in diesem Lande in Ewigkeit.

Das bedeutet, einen Mann zu unterweisen, um zur Nachwelt zu sprechen. Wenn er darauf hört, wird er zum Meisterhörer.

Gut ist es, zur Nachwelt zu sprechen: sie ist es, die darauf hören wird.

Wohltätig ist das Hören für den hörenden Sohn.

Wenn das Gehörte eintritt in den Hörenden, wird der Hörende zu einem, auf den gehört wird.

Wenn das Hören gut ist, ist das Reden gut, und der Hörende ist ein Besitzer von Wohltätigem.

Wohltätig ist das Hören für den Hörenden, besser ist Hören als alles andere, denn die Beliebtheit gelangt zur Vollendung. Wie schön ist, wenn ein Sohn annimmt, was sein Vater sagt: ihm wird hohes Alter zuteil dadurch; denn ein von Gott Geliebter ist der Hörende, aber ein von Gott Gehaßter kann nicht hören.

Es ist aber das Herz, das seinen Besitzer macht zu einem Hörenden oder zu einem Nicht-Hörenden. Leben, Heil und Wohlergehen eines Mannes ist sein Herz. Der Hörende hört das, was gesagt wird, der das Hören liebt, tut was gesagt wird.

Wie schön ist, wenn ein Sohn auf seinen Vater hört, wie froh ist der, zu dem gesagt solches wird. Der Sohn, er wird schön als ein Herr des Hörens, Ein Hörender, zu dem dies gesagt wird, er ist schon im Mutterleib wohltätig und grabversorgt, mehr als sein Vater.

Sein Andenken ist im Munde der Menschen, die jetzt auf Erden sind und derer, die sein werden.

Wenn der Sohn eines Mannes annimmt, was sein Vater sagt, dann schlägt keiner seiner Pläne fehl.

Du sollst deinen Sohn zu einem Hörenden erziehen, zu einem, der untadelig ist im Urteil der Notablen, einem, der seine Rede ausrichtet nach dem, was ihm gesagt wurde und der als ein Hörender angesehen wird.

Der Sohn, wenn er untadelig ist, ist seine Laufbahn ausgezeichnet, aber fehl schlägt die Einführung dem, der nicht hört. 
Der Tor aber, der nicht hört, für den wird nichts getan;

Wissen sieht er als Unwissen an, Förderliches als Schädliches:

Alles Schändliche tut er, so daß Klage geführt wird über ihn Tag für Tag.

Er lebt von dem, woran man stirbt, seine verderbliche Nahrung ist Sprechen.

Seine Verfassung darin ist den Fürsten bekannt, nämlich: lebendig tot zu sein Tag für Tag.

Man geht vorüber an seinen Notlagen

Wegen der Menge des ihm Widerfahrenden, Tag für Tag.

Zwei Punkte erscheinen mir an diesem Lob des Hörens besonders bemerkenswert. Der eine Punkt ist die Betonung der vertikalen, diachronen Achse dieser Kommunikation, das Hören auf die Vorfahren, das dazu befähigt, zu den Nachkommen zu sprechen. Ich sagte, dass die alten Ägypter in einer Welt ohne Post, Telefon und Internet lebten und daher auf das lebendige Zusammenleben angewiesen waren, um aufeinander hören zu können. Sie hatten aber die Schrift, die es möglich machte, den Horizont der Redner und Hörer in die Tiefe und Weite der Zeit auszudehnen. Der andere Punkt ist der enge Zusammenhang, in den Hören und Leben hier gebracht werden. Wer nicht hören kann, geht zugrunde. Leben ist Einbindung, und wer dazu unfähig ist, stirbt.

$\mathrm{Ma}$ 'at, so hatte ich sie definiert, ist der richtige Zusammenhang der Menschen und Dinge. In der Menschenwelt manifestiert sich der richtige Zusammenhang als Bindung und Zugehörigkeit des Einzelnen. Diese Zugehörigkeit, Bindung und Einbindung ist lebenspendend und lebensnotwendig, und sie wird durch sprachliche Kommunikation vermittelt. Der richtige Zusammenhang bedeutet nicht nur in der Sozialdimension Freundschaft und soziale Harmonie, sondern auch in der Zeitdimension Kontinuität und Bestand. Wer sich in die Ma'at, den richtigen $\mathrm{Zu}-$ sammenhang, einbindet, vergeht nicht. Ma'at ist das einzig Dauerhafte in dieser Welt, worauf sich der Mensch verlassen kann. So heißt es in demselben Literaturwerk, aus dem auch unsere Sentenz stammt, an anderer Stelle:

Ma'at aber wird ewig sein, sie steigt an der Hand dessen, der sie tat, ins Totenreich hinab.

Er wird begraben und vereint sich der Erde, aber sein Name wird nicht ausgelöscht werden auf Erden, sondern man gedenkt seiner wegen des Guten.

Das ist die Regel der Gottesworte. [...]

Sage die Ma'at, tue die Ma'at, denn sie ist groß und gewaltig; sie ist beständig, ihre Macht ist bewährt, sie allein geleitet zur Grabversorgtheit (jmibjj).

8 „Klagen des Bauern“, in: Assmann (1990), S. 113. 
Dieser Zusammenhang zwischen Ma'at, Gemeinschaft und Dauer wird auch deutlich, wenn wir einen Blick auf den unmittelbaren Kontext unserer Sentenz werfen. Sie erscheint dort als mittlerer von drei gleich gebauten Sätzen:

Die ganze Passage lautet ${ }^{9}$ :

Es gibt kein Gestern für den Trägen,

es gibt keinen Freund für den, der für die Ma'at taub ist,

es gibt kein Fest für den Habgierigen.

Die formale Struktur dieser drei Sätze ist ,es gibt kein A für B“, wobei unter A drei Formen von Gemeinschaftlichkeit gemeint sind und unter B drei Formen menschlicher Asozialität. So wie sich das Taubsein für die Ma'at auf das Fehlen von Sprache und Verständigung, so bezieht sich Trägheit auf das Fehlen von Handeln und Initiative. Wer nicht handelt, hat kein „Gestern", das heißt: keine Vergangenheit und keine Erinnerung. Wer nicht handelt, kann sich an nichts erinnern, und wer sich an nichts erinnern kann, ist ebenso aus der Gemeinschaft ausgeschlossen wie der Ma'at-Taube, der keinen Freund hat. Die Ausdrücke „Gestern“ und „Handeln“ kommen auch in einer Sentenz aus einem anderen Text derselben Zeit vor, in dem jemand über den Zerfall von Gemeinschaft, Gerechtigkeit und Ordnung klagt ${ }^{10}$ :

$\mathrm{Zu}$ wem kann ich heute noch reden?

Man erinnert sich nicht des Gestern, man handelt nicht für den, der gehandelt hat heutzutage.

Damit ist klar, was in unserem Text mit Trägheit gemeint ist: nicht einfach das Nicht-Handeln, sondern das Nicht-Handeln für andere, die für einen gehandelt haben, also der Zerfall der Reziprozität, des kommunikativen Handelns. Zum kommunikativen Handeln gehört Gedächtnis: dankbares Vergelten empfangener Wohltaten, korrektes Einhalten eingegangener Verpflichtungen, eben jenes „Gedächtnis des Willens", das sich nach Nietzsche die Menschen angezüchtet haben, um in Gemeinschaft leben zu können und das jeder Moral zugrunde liegt. Für die Ägypter schwingt in diesem Begriff des „Gestern“ aber auch der viel weitere Zeithorizont eines kommunikativen Handelns mit, der die Ahnen und damit auch die Nachkommen einbegreift.

9 Ebd., S. 60.

10 Aus dem „Gespräch eines Lebensmüden mit seinem Ba“, Papyrus Berlin 3024, in: ebd., S. 82-83. Diese drei Zeilen stehen im Text an herausgehobener Stelle. Sie bilden die mittlere Strophe eines ,anaphorischen Strophenliedes“, dessen Strophen alle mit dem Satz „Zu wem kann ich heute (noch) reden?" beginnen und das mit drei anderen Strophenliedern den ansonsten ,prosaischen" (oder besser gesagt: anders und schwächer geformten) Text lyrisch unterbricht. 


\section{II.}

Auch das Feiern-Können, zu dem der Habgierige unfähig ist, stellt eine Form von Gemeinschaftlichkeit dar. Der ägyptische Ausdruck für „Fest“ lautet wörtlich übersetzt „schöner Tag“, genau wie das hebräische yom tov oder jiddisch jonteff („schöner Tag") und bezieht sich genau wie das hebräisch-jiddische Gegenstück auf spontane, private Festlichkeiten im Gegensatz zum religiösen, kalendarisch festgelegten Fest. ${ }^{11}$ Diese Form des Feierns hat etwas mit Muße und Geselligkeit zu tun. Dazu ist der Habgierige außerstande. Ohne Handeln, so können wir die drei Sätze vielleicht zusammenfassen, kein Gedächtnis und keine Vergangenheit, ohne Sprache und Verständigung über die Ma'at keine Freundschaft, und ohne Altruismus, das Schenken-und-Teilen-Können, keine gesellige Muße.

Damit haben wir in diesen drei Sentenzen die Quintessenz dessen, was man mit einem Ausdruck Ekkehard Krippendorffs - die ägyptische Gemeinschaftskunst $^{12}$ nennen könnte, für die als herausragende Erscheinungsformen Vergangenheit, Freundschaft und gesellige Muße stehen und als deren herausragende anthropologische Grundvoraussetzungen bzw. Tugenden Tätigkeit, Zuhören und Altruismus genannt werden: füreinander handeln, aufeinander hören und miteinander feiern. Umgekehrt werden Nichthandeln, Nichthören und Habgier als die fundamentalen Sünden gegen die Gemeinschaft herausgestellt. Nichthandeln zerstört die Erinnerung, Nichthören zerstört die Freundschaft und Habgier zerstört das gesellige Feiern, die festliche Muße.

Die drei Sätze stellen die ägyptische Antwort dar auf die Frage, was die Menschen zur Gemeinschaft verbindet. Auch den ägyptischen Begriff der Freundschaft hat man im Zusammenhang dieser Frage nach den Bedingungen der Möglichkeit von Gemeinschaft zu verstehen. Es geht, wie schon gesagt, nicht um die private Beziehung zwischen zwei Menschen, sondern um die Bindekräfte, die ein ganzes Volk in Raum und Zeit zusammenhalten. In Ägypten führt das Thema „Freundschaft" wie bei Aristoteles sogleich auf die höhere Ebene der Gemeinschaft, und es bleibt auch auf dieser Ebene nicht stehen, wie ich im Folgenden zeigen will. Oberhalb dieser Ebene steht die Ebene des Staates und über dieser wiederum die Ebene des Kosmos. Der Einzelne hat sich in die Gemeinschaft, die Gemeinschaft in den Staat und der Staat in den Kosmos einzubinden. Dann ist das verwirklicht, was der Ägypter unter Ma'at versteht, für die empfänglich zu sein die Grundbedingung der Freundschaft darstellt. Ma'at ist nichts anderes als das Prinzip der Einbindung: des Einzelnen in die Gemeinschaft, der Gemeinschaft in den Staat und des Staats in den Kosmos.

Die lebenswichtige Bedeutung von Kommunikation und Zugehörigkeit, die ein Hauptthema der ägyptischen Literatur darstellt, ist von der modernen Wis-

11 Vgl. hierzu Jan Assmann, „Der schöne Tag. Sinnlichkeit und Vergänglichkeit im altägyptischen Fest", in: Das Fest, hrsg. von Walther Haug, Rainer Warning, München 1989, S. 3-28, ND in: Jan Assmann, Stein und Zeit. Mensch und Gesellschaft im Alten Ägypten, München 1991, S. 200-237.

12 Vgl. Anm. 5. 
senschaft, insbesondere der Hirnforschung und Psychologie, glänzend bestätigt worden und hat sich in verschiedenen Richtungen der psychotherapeutischen Praxis vielfältig bewährt. ${ }^{13}$ Besonders nah sind dabei jene Richtungen dem ägyptischen Menschenbild gekommen, die den Klienten nicht als monadisches Individuum, sondern als Glied in einem Beziehungsnetz verstehen, wie vor allem die Methode der System- oder Familienaufstellung. ${ }^{14}$ Die verblüffenden Wirkungen dieser Praxis sind bisher theoretisch noch kaum eingeholt und verstanden, was gewiss damit zusammenhängt, dass sie im Rahmen einer Gesellschaft entwickelt wurden, die einerseits den abendländischen Sonderweg des ,autonomen Selbst ${ }^{\text {“ }}$ und eines übersteigerten Individualismus beschritten hat, ${ }^{15}$ und andererseits nach zwei Weltkriegen und dem Holocaust von der Vergangenheit in einem bislang ungekannten Ausmaß heimgesucht wurde. ${ }^{16} \mathrm{Da}$ ich mich auf diesem Gebiet wenig auskenne, kann ich nicht beurteilen, inwieweit der Begriff des Systems bzw. der Zugehörigkeit neben verwandtschaftlichen Beziehungen auch freundschaftliche Bindungen umfasst. Im Alten Ägypten jedenfalls ist diese Ausweitung über das Genetisch-Biologische hinaus auf das Kulturelle, Geistige ganz eindeutig. Die Bindungen, auf die es ankommt, werden nicht nur durch die Geburt in eine Familie hinein, sondern durch kommunikative Sozialisation in eine Gemeinschaft und einen Staat gestiftet.

Diese Unterscheidung zwischen dem Genetischen und dem Epigenetischen wurde sogar bereits von den Alten Ägyptern gemacht. Sie sprachen dem Menschen zwei Herzen zu: ein Herz von der Mutter, also ein angeborenes Herz, und ein Herz von dem Dasein auf Erden, also ein erworbenes Herz. ${ }^{17}$ Das eine Herz nannten sie $j b$, was mit dem hebräischen Wort leb/lebab verwandt ist, das andere hati, was mit dem ägyptischen Wort für „vorn“, „Vorderseite“, „Brust“ und „Stirn“ zusammenhängt. Da sie das Herz meist mit dem Bildzeichen „Herz“, also ideo- und nicht phonographisch schreiben, wissen wir meist nicht, welches Herz gemeint ist. Auf jeden Fall aber ist das Herz das Organ des Hörens. Das Ohr nimmt nur die Töne auf, das Herz verarbeitet sie in Sprache und verständlichen Sinn. Dieser Sinn ist es, den der Mensch zum Leben braucht. Hören wir noch einmal die Worte des Ptahhotep über das hörende Herz ${ }^{18}$ :

13 Vgl. etwa die Forschungen von Tania Singer (Leipzig), Joachim Bauer (Freiburg) und Nils Birbaumer (Tübingen).

14 Vgl. hierzu von a. die Publikationen von Gunthard Weber (Heidelberg).

15 Die autonome Person - eine europäische Erfindung?, hrsg. von Klaus-Peter Koepping, Michael Welker und Reiner Wiehl, München 2002.

16 Vgl. hierzu Aleida Assmann, Der lange Schatten der Vergangenheit. Erinnerungskultur und Geschichtspolitik, München 2006.

17 Vgl. Hellmut Brunner, „Das Herr im ägyptischen Glauben“, in: Das Herz im Umkeris des Glaubens, Biberach 1965, S. 81-106; Jan Assmann, „Zur Geschichte des Herzens im alten Ägypten“, in: Die Erfindung des Inneren Menschen. Studien zur religiösen Anthropologie, hrsg. von Jan Assmann und Theo Sundermeier, Gütersloh 1993, S. 81-112.

18 Ptahhotep Dévaud 534-536, in: Assmann (1990), S. 76. 
Es ist aber das Herz, das seinen Besitzer macht zu einem Hörenden oder zu einem Nicht-Hörenden.

Leben, Heil und Wohlergehen eines Mannes ist sein Herz.

Dass diese Frage im Alten Ägypten eine derart zentrale Rolle spielte, ist kein Wunder: haben wir es doch hier mit dem ersten Großstaat der Geschichte zu tun. Dieser Staat entstand in der Wende vom vierten zum dritten Jahrtausend und hatte für ca. 1000 Jahre Bestand, bis er dann am Ende des dritten Jahrtausends in einzelne Regionalherrschaften zerfiel. Erstaunlicher aber noch als diese frühe und so lang dauernde Gründung ist ihre „Resilienz", ihre Fähigkeit, sich auch nach Zusammenbrüchen wieder zu reorganisieren. ${ }^{19}$ Nach einem 100 - bis 150-jährigen Intervall ohne Zentralkönigtum gelang es den thebanischen Fürsten, ihre Konkurrenten zu besiegen und die pharaonische Monokratie wieder zu errichten. Aus dieser Zeit stammen die Texte, die über die Grundlagen von Staat und Gemeinschaft reflektieren. Jetzt wird nach tausendjähriger politischer Praxis die zugehörige Theorie nachgeliefert und explizit gemacht. Das zentrale Thema ist „Bindung“. Wie gelingt es dem Einzelnen, sich einzubinden in die Gemeinschaft, deren politische Form der pharaonische Staat darstellt? So entsteht eine Ideologie der Einbindung, die sich in zwei Kernthesen zusammenfassen lässt: Der Einzelne ist nichts, solange er sich nicht in den verschiedenen Konstellationen des gemeinschaftlichen Lebens, Familie, Nachbarschaft, Stadtgemeinschaft, bis hinauf zur höchsten Form der Einbindung, dem Königsdienst, engagiert, und diese Einbindung, das ist die zweite These, ist ohne Staat, d. h. das pharaonische Zentralkönigtum, nicht möglich. Beide Thesen haben eine unverkennbar totalitäre, antiindividualistische Tendenz. Die erste These erinnert fatal an den Satz „Du bist nichts, das Volk ist alles“, den man im NS-Deutschland ständig zu hören bekam, und die zweite These entspricht der damals herrschenden Vorstellung, dass auch das Volk nichts ist ohne seinen Führer. Haben wir es beim pharaonischen Staat mit einer Frühform von Totalitarismus zu tun?

Hier muss zunächst einem naheliegenden Missverständnis entgegengetreten werden. Ma'at ist das Prinzip der Konnektivität. Konnektivität aber ist etwas anderes als Kollektivität. Kollektivität wäre die Unterordnung des Individuums unter das Kollektiv. Kollektivität bzw. Kollektivismus wirkt von oben nach unten. Vorrangig gegenüber dem Begriff des Individuums ist ein Begriff von Kollektiv wie ,Volk“, „Gemeinschaft", „Staat“ (im Sinne von polis oder res publica), „Religionsgemeinschaft" usw., dem sich der Einzelne unterzuordnen hat. Solche Kollektivbegriffe fehlen aber im Ägyptischen, in eklatantem Gegensatz zu Israel, wo der Begriff des Volkes nicht nur mit zwei Lexemen ( $\mathrm{c}$ am und goj) realisiert, sondern durch den des auserwählten oder heiligen Volkes noch emphatisch gesteigert ist. Konnektivität dagegen wirkt von unten nach oben; sie ist eine Sache konnektiver Tugenden, die das Individuum befähigen, sich in kleinere und gröBere Konstellationen und Kreise, von der Familie bis hinauf zum Königsdienst einzubinden. In dieser Konnektivität erblickt der Ägypter das Geheimnis des Le-

19 Vgl. hierzu Jan Assmann, Agypten. Eine Sinngeschicbte, Frankfurt a. M. 2000. 
bens. Leben heißt: Bindungen eingehen und aufrecht erhalten. Tod ist Auflösung, Vereinzelung, Vereinsamung. Ein ägyptisches Sprichwort lautet: „Einer lebt, wenn ein anderer ihn leitet; "20 es bedeutet dasselbe wie das afrikanische Sprichwort „nur gemeinsam können wir leben“21. Erst wenn man den Unterschied zwischen Konnektivität und Kollektivität berücksichtigt, darf man in Bezug auf das Alte Ägypten von „Staat" und „Gemeinschaft" sprechen.

Angesichts dieser Thematik liegt es nahe, sich an Carl Schmitt und seine berühmt-berüchtigte Schrift über den Begriff des Politischen zu wenden, in der es ja genau um die Frage nach dem Prinzip der Assoziation geht, dem Prinzip, nach dem, wie es mehrfach heißt, ,sich die Völker gruppieren“22. Schmitts bekannte Antwort lautet: Die Völker gruppieren sich nach dem Kriterium von Freund und Feind. Auch hier stoßen wir also auf das Motiv der Freundschaft, allerdings eingebunden in eine dialektische Beziehung zur Feindschaft. Ich finde es lohnend, Schmitts diesbezügliche Ausführungen einmal im Licht der altägyptischen Befunde zu betrachten. Wo bleibt eigentlich der Feind in der ägyptischen Gemeinschaftslehre? Wenn auf politischer Ebene Assoziation und Dissoziation dialektisch aufeinander bezogen sind, es hier also keine Freundschaft ohne Feindschaft geben kann, weil nur der gemeinsame Feind in der Lage ist, die Menschen als Freunde zur Gemeinschaft zu verbinden, dann müsste sich doch auch in den altägyptischen Reflexionen über Freundschaft und Gemeinschaft der Schatten des gemeinsamen Feindes abzeichnen. Hören wir zunächst Carl Schmitt.

Schmitt nähert sich dem Begriff des Politischen mit einer Methode, die stark an Niklas Luhmann erinnert. Er fragt nämlich nach der spezifischen Unterscheidung, die dem Politischen als einem kulturellen Gebiet zugrunde liegt und es gegenüber anderen Gebieten identifiziert. Luhmann spricht in diesem Zusammenhang von „Leitunterscheidungen“, Schmitt von „letzten Unterscheidungen“ "23:

Nehmen wir an, daß auf dem Gebiet des Moralischen die letzten Unterscheidungen Gut und Böse sind; im Ästhetischen Schön und Häßlich; im Ökonomischen Nützlich und Schädlich oder beispielsweise Rentabel und Nicht-Rentabel. Die Frage ist dann, ob es auch eine besondere, jenen anderen Unterscheidungen zwar nicht gleichartige und analoge, aber von ihnen doch unabhängige, selbständige und als solche ohne weiteres einleuchtende Unterscheidung als einfaches Kriterium des Politischen gibt und worin sie besteht.

20 In einem magischen Text äußert die Göttin Isis diese Sentenz wie ein gängiges Sprichwort. Metternichstele M 50, vgl. Constantin-Emil Sander-Hansen, Die Texte der Metternichstele, Kopenhagen 1956, S. 35-36, 41; Adolf Klasens, A Magical Statue Base (Socle Behague) in the Museum of Antiquities at Leiden, Leiden 1952, S. 10 und 52; Heike Sternberg, „Die Metternichstele“, in: Texte aus der Umvelt des Alten Testaments (TUAT), hrsg. von Otto Kaiser, Bd. II.3 (Rituale und Beschwörungen II), Gütersloh 1988, S. 376.

21 Theo Sundermeier, Nur gemeinsam können wir leben. Das Menschenbild schwarzafrikanischer Religionen, Gütersloh 1988.

22 Carl Schmitt, Der Begriff des Politischen. Text von 1932 mit einem Vonvort und drei Corollarien, unveränderter ND der 1963 erschienenen Auflage, Berlin 1979; hier z. B. S. 29.

23 Ebd., S. 26-27. 
Die spezifisch politische Unterscheidung, auf welche sich die politischen Handlungen und Motive zurückführen lassen, ist die Unterscheidung von Freund und Feind. Sie gibt eine Begriffsbestimmung im Sinne eines Kriteriums, nicht als erschöpfende Definition oder Inhaltsangabe. Insofern sie nicht aus anderen Kriterien ableitbar ist, entspricht sie für das Politische den relativ selbständigen Kriterien anderer Gegensätze: Gut und Böse im Moralischen; Schön und Häßlich im Ästhetischen usw. Jedenfalls ist sie selbständig, nicht im Sinne eines eigenen neuen Sachgebiets, sondern in der Weise, daß sie weder auf einem jener anderen Gegensätze oder auf mehreren von ihnen begründet, noch auf sie zurückgeführt werden kann.

Doch bleibt Schmitt bei dieser gewissermaßen systemtheoretischen Ebene nicht stehen, auf der das Politische sich als ein kulturelles Subsystem sui generis identifizieren lässt. Die Unterscheidung von Freund und Feind dient Schmitt zwar dazu, das Politische als ein autonomes ,geistiges Gebiet“ zu identifizieren, aber dann will er dieses Gebiet gerade nicht gleichberechtigt neben die anderen stellen, sondern ihnen überordnen. Mit seiner Unterscheidung von Freund und Feind plädiert Schmitt nicht für die Autonomie, sondern für die Hegemonie des Politischen. „Das Politische“, schreibt Schmitt ${ }^{24}$,

kann seine Kraft aus den verschiedensten Bereichen menschlichen Lebens ziehen, aus religiösen, ökonomischen, moralischen und anderen Gegensätzen; es bezeichnet kein eigenes Sachgebiet, sondern nur den Intensitätsgrad einer Assoziation oder Dissoziation von Menschen, deren Motive religiöser, nationaler, wirtschaftlicher oder anderer Art sein können [...]. Die reale Freund-Feind-Gruppierung ist seinsmäßig so stark und ausschlaggebend, daß der nichtpolitische Gegensatz [...] seine bisherigen „rein" religiösen, „rein" kulturellen Kriterien und Motive zurückstellt [...]. Politisch ist jedenfalls immer die Gruppierung, die sich an dem Ernstfall orientiert. Sie ist deshalb immer die maßgebende menschliche Gruppierung.

Der entscheidende Begriff in dieser Argumentation scheint mir der Begriff des „Ernstfalls“. Erst im Licht des Ernstfalls konstituiert sich das Politische nicht nur als ein eigenes, sondern ein totalisierendes, alle anderen kulturellen Gebiete dominierendes Sachgebiet. Der Ernstfall ist der Kriegsfall. So könnte man annehmen, dass für Schmitt das Politische überhaupt nur im Krieg zutage tritt. Erst im Krieg würde dann gelten, „daß die Völker sich nach dem Gegensatz von Freund und Feind gruppieren." Das will Schmitt aber nicht sagen. Dieser Gegensatz gilt für ihn vielmehr immer und überall. Daher führt er den Begriff der „realen Möglichkeit “ ein und fügt hinzu: „Daß dieser Gegensatz auch heute noch wirklich und für jedes politisch existierende Volk als reale Möglichkeit gegeben ist, kann man vernünftigerweise nicht leugnen." 25 Die ,reale Möglichkeit der Gruppierung von Freund und Feind“, schreibt Schmitt, ,genügt, um über das bloß Gesellschaftlich-Assoziative hinaus eine maßgebende Einheit zu schaffen, die etwas spezifisch anderes und gegenüber den übrigen Assoziationen etwas Entscheidendes ist. “ 26

24 Ebd., S. 38-39.

25 Ebd., S. 29.

26 Ebd., S. 45 
Unter einer „realen Möglichkeit“ versteht Schmitt eine Situation, die zwar nicht vorliegt, mit deren Eintreten man aber so sicher rechnen kann, dass man die gesamte politische und gesellschaftliche Verfassung darauf einrichten muss. Daher gruppieren sich die Menschen auch in Friedenszeiten und ohne das Auftreten irgendeines Feindes nach dem Gegensatz von Freund und Feind. Das Leben gewinnt seine Ordnungen und seinen Sinn nicht vom Alltag her, sondern vom Ernstfall. Mit seinen Kategorien des Ernstfalls und seiner realen Möglichkeit steht Schmitt ganz im Bann des Zeitgeists der 20er und 30er Jahre, des Expressionismus und Existenzialismus. Unter den Bedingungen des Ernstfalls wird das Politische das Totale; dabei bleibt aber Carl Schmitt nicht stehen, sondern er begründet darauf den Anspruch, das Politische schlechthin, auch unter normalen Bedingungen, zum Totalen zu erklären, da ja der Ernstfall als Möglichkeit immer gegeben ist. Zu den nie wirklich explizit gemachten Grundsätzen seiner Lehre gehört der fraglose Vorrang der Ausnahme vor der Regel. ${ }^{27}$

Das Politische erschöpft sich also für Schmitt nicht in der Frage der Assoziation und Dissoziation, der Gruppen-, Nationen- und Staatenbildung, der Zugehörigkeit und Nichtzugehörigkeit, sondern es transzendiert diese Ebene der Vergesellschaftung. „In Wahrheit gibt es keine politische ,Gesellschaft‘ oder ,Assoziation', es gibt nur eine politische Einheit, eine politische ,Gemeinschaft "“c28. Im Ernstfall, Jaspers würde sagen, in der „Grenzsituation“, des Krieges offenbart sich, was normalerweise verhüllt bleibt: dass sich die Menschen nach Freund und Feind gruppieren. Daher ist für Schmitt der Ausnahmezustand nicht die Umkehrung des Normalen, sondern seine Entlarvung, die Stunde der Wahrheit, genauso wie für den Existentialismus die Grenzsituation die Dinge nicht auf den Kopf stellt, sondern in ihrer wahren Bedeutung enthüllt. Wenn Heidegger das Leben als „Dasein zum Tode“ definiert, dann sieht er im Tod nicht das Gegenteil, sondern, mit Schmitt gesprochen, den ,Ernstfall“ des Lebens, dessen Bedeutung nur von diesem Ernstfall her erkannt werden kann. So wie Heidegger das Leben als Dasein zum Tode definiert Schmitt das Politische als Dasein zum Krieg, den er damit in den Rang eines Existenzials erhebt.

\section{III.}

Wenn wir nach diesem Exkurs über Carl Schmitt wieder zu den Alten Ägyptern zurückkehren, treten interessante Unterschiede zutage. Die ägyptische Staatenbildung vollzog sich in einer Frühzeit, in der Krieg und politische Feindschaft nicht ins Blickfeld der politischen Reflexion traten. Die Menschen gruppierten sich hier nach anderen Kriterien. Zwar vollzog sich der Prozess der Reichseinigung, wenn

${ }^{27}$ Was für die Feindschaft gilt, gilt auch für die Freundschaft. Wie man im Hinblick auf den Ernstfall wissen muss, wer der Feind ist, und bereit sein muss, ihn zu töten, muss man auch den Freund kennen und bereit sein, für ihn zu sterben. Schillers Ballade Die Bürgschaft handelt vom Ernstfall der Freundschaft.

28 Schmitt (1979), S. 45. 
man den zeitgenössischen Darstellungen glauben darf, nicht ohne Gewalt, sondern in der Form eines Sieges des Südens über den Norden, aber das war kein Krieg, der Norden war kein Feind, sondern ein integraler Teil des sich gründenden Reiches. Die Geschichte von Horus und Seth fasst diese Vorgänge im Medium einer mythischen Erzählung. ${ }^{29}$

Im Anfang, so ließe sich dieser Mythos vielleicht zusammenfassen, war der Sonnengott, aus dem alles hervorging, Zeit und Raum, Himmel und Erde, Götter und Menschen, Tiere und Pflanzen. Was aus dem Gott entstand, bleibt von seinem Ursprung abhängig, und diese Abhängigkeit deuten die Ägypter als Herrschaft, die also zugleich mit der Welt entsteht. Zuerst übte der Sonnengott, aus dem alles entstand, diese Herrschaft selbst aus, dann delegierte er sie an seinen Sohn, den Luftgott Schu, von dem sie auf den Erdgott Geb überging. Die Herrschaft beschreibt also eine Abwärtsbewegung, vom Himmel durch den Luftraum auf die Erde. Wichtig ist vor allem, dass sie sich aus der Schöpfung selbst herleitet. Geb aber hatte zwei Söhne: Osiris und Seth. Er überwies die Herrschaft dem Osiris, aber dieser wurde von seinem Bruder und Rivalen Seth erschlagen, der die Herrschaft an sich reißen wollte. Hier stoßen wir also auf das Thema Feindschaft, und zwar ganz im Schmittschen Sinne. Mit Seth gewinnt die Herrschaft ihren Feind, gegen den sie sich durchzusetzen hat. Das ist die Aufgabe des Horus, des nachgeborenen Sohnes, den Isis ihrem erschlagenen Gatten schenkt. Der zieht, kaum herangewachsen, den Mörder und Usurpator vor Gericht und erreicht, dass er selbst als Nachfolger seines Vaters eingesetzt wird. Mit Horus tritt die Herrschaft in die Geschichte ein, denn nach ägyptischer Vorstellung verkörpert sich dieser Gott in jedem menschlichen König. Jetzt aber geschieht das Außerordentliche: Seth akzeptiert das Urteil und die streitenden „Brüder“, wie es ägyptisch heißt (eigentlich handelt es sich ja um Onkel und Neffe wie in Shakespeares Hamlet), versöhnen sich. Dieser Akt der Versöhnung ist das zentrale Symbol der Reichsgründung. Auf den Seiten des Königsthrons ist dargestellt, wie Horus und Seth Lotus und Lilie, die Wappenpflanzen von Ober- und Unterägypten, zusammenbinden. Seth ist durchaus der Feind des Horus und in allem dessen genaues Gegenstück. Horus steht für Recht, Ordnung und Legitimität, Seth für rohe Brachialgewalt. Seth verkörpert den Gegensatz aller Tugenden, die die Menschen zum Leben in Gemeinschaft befähigen. Von Seth heißt es in einem späten Ritualtext: „Der über Trennung zufrieden ist und Verbrüderung haßt, der sich (nur) auf sein (eigenes) Herz stützt unter den Göttern." ${ }^{\text {" }}$

Dieser Text stammt aber aus einer Zeit, in der sich die ägyptische Welt und mit ihr die Gestalt des Gottes Seth gründlich gewandelt hatte. Jetzt ist Seth wirklich zum providentiellen Feind im Sinne Carl Schmitts geworden. Ägypten hat den Ernstfall kennengelernt in Gestalt mehrfacher Eroberungen und Fremdherrschaften, und Seth trägt jetzt den Beinamen „der Meder“. Er ist nun zu einer Art

29 Vgl. hierzu Assmann (2000).

30 Urkunden mythologischen Inhalts VI, hrsg. von Siegfried Schott, Leipzig 1929-1939, 7.15-16; in der Folge zitiert als Urk VI. 
Teufel geworden, bei dem jede Form der Integration in die ägyptische Welt undenkbar ist. In der älteren Zeit dagegen wird er bei all seiner mörderischen und chaotischen Gewalttätigkeit in diese Welt integriert, weil auch das Recht der Gewalt bedarf und die Ma'at nicht ohne Gewalt durchzusetzen ist. Das Recht bedarf, wie es schon Blaise Pascal in einer berühmten Passage seiner Pensées feststellte, der Gewalt, um in Kraft zu treten ${ }^{31}$ :

Die Gerechtigkeit ohne Gewalt ist kraftlos, ohnmächtig; Gewalt ohne Gerechtigkeit ist tyrannisch. Gerechtigkeit ohne Gewalt wird nicht anerkannt, weil es immer Bösewichte gibt; Gewalt ohne Gerechtigkeit wird angeklagt. Man muß also Gerechtigkeit und Gewalt zusammenstellen, damit was gerecht und angemessen auch stark und kräftig, was stark und kräftig auch gerecht und angemessen ist. [...]

Da man nicht hat erreichen können, daß das Gerechte und Angemessene auch stark und kräftig ist, hat man dafür gesorgt, daß das Starke und Kräftige gerecht und angemessen ist.

Das heißt, man hat das Starke und Kräftige dem Gerechten und Angemessenen untergeordnet, und genau das ist die Zentralaussage des Mythos von Horus und Seth.

Seth ist also nicht der providentielle Feind, „die eigene Frage als Gestalt", wie es in dem von Schmitt so gern zitierten Vers von Theodor Däubler heißt ${ }^{32}$ :

Der Feind ist unsere eigne Frage als Gestalt

Und er wird uns, wir ihn zum selben Ende hetzen.

Nein, das Ende dieses Konflikts ist ganz anders, es ist eine Versöhnung, durch die erst das Ganze des Staats und der Gemeinschaft in seiner Vollständigkeit entsteht. Seth verkörpert nicht den Ernstfall der ägyptischen Welt, auf dessen reale Möglichkeit hin sie sich gruppiert und eingerichtet hat. Aber für diese Rolle gibt es noch einen anderen Kandidaten, und gegen den wird nun kein anderer als Seth als Retter aufgeboten. Der Sonnengott holt sich den im Streit um die Thronfolge des Osiris Unterlegenen als Beistand in seine Barke, in der er tags über den Himmel und nachts durch die Unterwelt fährt, damit er den Apophisdrachen bekämpft. Apophis ist der providentielle Feind der ägyptischen Welt, er verkörpert den Ernstfall, auf den hin als eine reale Möglichkeit sich die Ägypter zusammenschließen. Dieser Ernstfall ist nicht der Krieg, sondern eine kosmische Katastrophe, die entsteht, wenn es Apophis gelingt, den Himmelsozean auszusaufen, sodass die Sonnenbarke auf Grund läuft und nicht mehr weiterfahren kann. Angesichts dieses Feindes wird selbst Seth, der Raufbold, Mörder und Usurpator, zum Freund.

31 Blaise Pascal, Pensées et opuscules, hrsg. von Léon Brunschvicg, Paris 1912, S. 470 (Nr. 298), zit. nach Jacques Derrida, Gesetzeskeraft. Der mystische Grund der Autorität, Frankfurt a. M. 1991, S. 23.

32 Theodor Däubler, „Sang an Palermo“, in: ders., Hymne an Italien, Leipzig 1919, S. 65; vgl. Heinrich Meier, Carl Schmitt, Leo Strauss und der „Begriff des Politischen“. Zu einem Dialog unter Abwesenden, Stuttgart 1998, S. 91, Anm. 103 sowie ders., Die Lebre Carl Schmitts. Vier Kapitel zur Unterscheidung politischer Theologie und politischer Philosopbie, Stuttgart 1994, S. 76 ff. 
Im Licht von Schmitts Bestimmung des Politischen erweist sich die ägyptische Welt als vor-politisch. Apophis ist kein politischer, sondern ein kosmischer Feind. Zwischen dem Politischen und dem Kosmischen herrscht eine enge Beziehung, kommt es doch in Ägypten nicht nur darauf an, die Menschen zu Gemeinschaft und Staat zu verbinden, sondern diesen Staat in den Kosmos einzubinden. Die politische Herrschaft legitimiert sich im ägyptischen Denken vom Ursprung her. „Entstehen aus“ bedeutet „Abhängigkeit von“, und „Abhängigkeit von“ bedeutet „Herrschaft über“. Da nach ägyptischer Vorstellung alles aus der Sonne entstanden ist, ist auch alles von der Sonne abhängig, was bedeutet, dass die Sonne über alles herrscht. Diese Herrschaft übt der Sonnengott auf kosmischer Ebene durch seine Barkenfahrt aus, auf Erden aber hat er Pharao als seinen Sohn und Stellvertreter eingesetzt ${ }^{33}$ :
RE hat den König N. N. eingesetzt
auf der Erde der Lebenden
für unendliche Zeit und unwandelbare Dauer
beim Rechtsprechen den Menschen, beim Zufriedenstellen der Götter, beim Verwirklichen der Wahrheit, beim Vernichten der Sünde (jsft); er gibt den Göttern Opferspeisen, und den Verklärten Totenopfer.

Der König verwirklicht die Ma'at durch Recht und Kult: Indem er den Menschen Recht spricht und den Göttern und Toten Opfer darbringt. In allen Sphären stellt die Verwirklichung der Ma'at Zusammenhang und Gemeinschaft her. Der Kult sorgt für den Einklang von Himmel, Erde und Totenreich, und das Recht sorgt für Gemeinschaft und Ordnung unter den Menschen. Den Menschen Recht zu sprechen heißt, wie wir aus anderen Quellen erfahren, zu ,richten zwischen dem Schwachen und dem Starken“" ${ }^{\text {34. }}$

\section{IV.}

„Richten“ bedeutet in erster Linie „schlichten“. Dabei geht es nicht um die Vollstreckung von Gesetzen einer zeitenthobenen Gerechtigkeit, sondern um die Wiederherstellung von Harmonie und Gemeinschaft durch die Schlichtung von Streit und Zwietracht. Das Ideal des guten Richters ist Thot, der Gott, „der die streitenden Brüder (Horus und Seth) trennte, so dass beide zufrieden waren" 35 . Der letzte Zweck des Urteils besteht nicht in der Bestrafung des Übeltäters, sondern in der Wiederherstellung der gestörten Harmonie.

33 Jan Assmann, Der König als Sonnenpriester. Ein kosmographischer Begleittext zur kultischen Sonnenbymnik in thebanischen Tempeln und Gräbern, Stuttgart u. a. 1970; ders., Sonnenbymnen in Thebaniscben Gräbern, Mainz 1983, S. 48-49; Assmann (1990), S. 205-212; Maria C. Betrò, I testi solari del portale di Pascerientaisu, Pisa 1989.

34 Assmann (1990), S. 213-217.

35 Urle VI. 
Der Begriff der Isfet bezeichnet den Zustand des Gegensatzes menschenweltlicher Gerechtigkeit zu götterweltlicher Harmonie, dem durch Rechtsprechung und Kult abgeholfen werden muss. Isfet bedeutet aber nicht einfach den Mangel an (die Abwesenheit von) Gerechtigkeit und Harmonie, sondern deren Gegenteil, d. h. Unrecht und Gewalt. Das ist entscheidend. Die „Erde der Lebenden“, auf der der König durch Rechtsprechung und Kult die Ma'at verwirklichen soll, ist keine tabula rasa, sondern eine Sphäre, in der vorgängig, gewissermaßen von $\mathrm{Na}$ tur aus, Isfet herrscht. Der König muss die Isfet vernichten, um die Ma'at zu verwirklichen. In der polarisierten Welt ist jeder Akt der Ma'at-Verwirklichung ein Akt der Isfet-Vernichtung - und umgekehrt. In den Klagen des Oasenmannes wird diese dialektische Beziehung in eindrucksvollen Bildern zum Ausdruck gebracht ${ }^{36}$ :

Wer die Lüge vernichtet, fördert die Ma'at, wer das Gute fördert, macht das Böse zunichte, wie Sattheit den Hunger vertreibt, Kleidung den Nackten bedeckt, wie der Himmel heiter ist nach heftigem Sturm, daß er alle Frierenden wärme, wie Feuer rohe Nahrung kocht, wie Wasser den Durst löscht.

Die Lehre von der Isfet ist entscheidend für das Verständnis der ägyptischen Staatstheorie: denn auf ihr beruht die Auffassung von der Notwendigkeit des Staates. Isfet bezeichnet nicht einfach einen Mangel, dem abgeholfen werden muss, sondern eine gegenstrebige Kraft des Bösen, gegen die das Gute - die Ma'at - durchgesetzt und immer wieder aufrechterhalten werden muss durch unablässige Gegensteuerung gegen die Gravitation zum Bösen. Diese Gegensteuerung besteht in Rechtsprechung und Kult.

\section{V.}

Im letzten Teil dieses Beitrags möchte ich der ägyptischen Konstruktion von Freundschaft, Feindschaft und Gemeinschaft die biblischen, d. h. alttestamentlichen Antworten auf die Frage gegenüberstellen, was die Menschen zur Gemeinschaft verbindet und nach welchen Kriterien sie sich gruppieren. Die biblische Geschichte unterscheidet hier drei Phasen. Die erste Phase reicht von der Schöpfung bis zu Abraham. Hier bewegen wir uns genau wie in Ägypten im Raum des Vor-Politischen. Völker gibt es noch nicht, es gibt nur Menschen, die aufgrund ihrer Schlechtigkeit von Gott durch die Sintflut wieder vernichtet werden mit Ausnahme des Noah, seiner Familie und seiner Archenbesatzung, mit denen Gott einen neuen Anfang machen will. Dieser mit Noah geschlossene Bund gilt der ganzen Menschheit. Auch der Turmbau von Babel ist noch ein Menschheits-

36 „Klagen des Bauern“, in: Erik Hornung, Altägyptische Dicbtung, Stuttgart 1996, S. $20-21$. 
projekt, und die Trennung der Sprachen bedeutet noch keine politische Trennung. Erst mit der Auswanderung des Abraham aus Harran beginnt eine neue Phase, die Gott dadurch besiegelt, dass er mit Abraham einen neuen Bund schließt, der nun nicht mehr für die ganze Menschheit, sondern nur für ihn und seine Nachkommen gilt und an die Beschneidung als das "Zeichen des Bundes“ geknüpft ist. Damit wird eine Differenz markiert, die aber zunächst noch keinen politischen Charakter hat und keine Gemeinschaft stiftet, da es sich ja nur um eine Familie handelt, die denn auch mehr oder weniger friedlich mit ihren kanaanitischen, hethitischen und ägyptischen Gastländern zusammenlebt. Immerhin aber ist von jetzt an von Völkern und Fremden die Rede. Die dritte Phase beginnt mit dem 2. Buch Mose, dem Buch Exodus. Jetzt ist aus der Familie ein Volk geworden, das sich durch Absetzung von und Auswanderung aus Ägypten, also durch einen Akt der Sezession, identifiziert, bei dem Ägypten durchaus die Rolle des Feindes im Schmittschen Sinne spielt. Hier endet der Konflikt nicht in Versöhnung, sondern in Rettung (Israel) und Vernichtung (Ägypter). Erst diese dritte Phase lässt sich als die im eigentlichen Sinne politische Phase bestimmen. Das Volk Israel gruppiert sich vollkommen eindeutig im Schmittschen Sinne nach dem Gegensatz von Freund und Feind, der als Unfreiheit vs. Freiheit, Despotie vs. Gesetz bestimmt wird, wobei das Sklavenhaus Ägypten als Inbegriff der Unfreiheit und der Raum des Gesetzes, der im Gelobten Land errichtet werden soll, als Inbegriff der Freiheit erscheinen. Das entscheidende Sammlungszeichen und Kriterium, nach dem sich das Volk zur Gemeinschaft verbindet, ist aber das Gesetz, die Thora, die ihm durch Mose am Sinai gegeben wird und die hier genau dieselbe Rolle spielt wie die Ma'at in Ägypten. Auch hier kommt alles darauf an, für die Thora nicht taub zu sein, sondern sie in Lektüre, Gespräch und Unterweisung unablässsig zu kommunizieren. „Du sollst sie deinen Söhnen wiederholen. Du sollst von ihnen reden, wenn du zu Hause sitzt und wenn du auf die Straße gehst, wenn du dich schlafen legst und wenn du aufstehst ${ }^{{ }^{*}{ }_{37}}$. Der entscheidende Unterschied zwischen der israelitischen Thora und der ägyptischen Ma'at liegt darin, dass es sich bei der Thora um eine politische Verfassung handelt, die nicht der Menschheit, sondern dem Volk Israel als Vertragsgrundlage seines Bündnisses mit Gott gegeben ist und eine Differenz zu den anderen Völkern markiert, während die Ma'at keinen Differenz- und damit auch keinen politischen Charakter hat und keine schriftlich fixierte Verfassung darstellt, sondern einen ständig zu erweiternden und neuen Erfahrungen anzupassenden Vorrat von Erfahrungswissen, eine zwar schriftlich artikulierbare, aber niemals festzuschreibende Gemeinschaftskunst, von der es immer heißt, dass sie „auf Erden“ und nicht etwa nur für Ägypten gilt.

In der biblischen Geschichte gibt es nun auch einen geradezu paradigmatischen Kandidaten für die Rolle des providentiellen Feindes, der eigenen Frage als Gestalt. Das ist Amalek, der Feind, den Israel besiegen konnte, solange Mose

37 Dtn 6,7; vgl. dazu auch Dtn 11,20. Die Bibelzitate beziehen sich in der Folge auf Die Bibel. Altes und Neves Testament. Einbeitsübersetzung, Freiburg u. a. 1980. 
seine Arme hoch hielt, wonach der Herr zu Mose sagte: „Halte das zur Erinnerung in einer Urkunde fest und präg es Josua ein! Denn ich will die Erinnerung an Amalek unter dem Himmel austilgen." ${ }^{38}$ Im Deuteronomium wird die Schlacht ganz anders erzählt, aber auch diese Erzählung schließt mit dem paradoxen Gebot, immer daran zu denken, dass Amalek vergessen werden muss ${ }^{39}$ :

\begin{abstract}
Denk daran, was Amalek dir unterwegs angetan hat, als ihr aus Ägypten zogt: wie er unterwegs auf dich stieß und, als du müde und matt warst, ohne jede Gottesfurcht alle erschöpften Nachzügler von hinten niedermachte. Wenn der Herr, dein Gott, dir von allen deinen Feinden ringsum Ruhe verschafft hat in dem Land, das der Herr, dein Gott, dir als Erbbesitz gibt, damit du es in Besitz nimmst, dann lösche die Erinnerung an Amalek unter dem Himmel aus! Das sollst du nicht vergessen.
\end{abstract}

Amalek ist durch diese Auslöschung der Erinnerung als der providentielle Feind Israels unsterblich geworden. Die hebräische Inschrift auf dem Pariser Mahnmal des unbekannten jüdischen Märtyrers zitiert Dtn 25,17 - „Denk daran, was Amalek dir angetan hat“ - und fährt fort: ,der Amalek unserer Tage, der sechs Millionen Leben - Körper und Seelen - auslöschte. “40 Mit dem Auszug aus Ägypten und der Landnahme in Kanaan tritt die biblische Geschichte in ihre politische Phase ein. Das Volk findet seine Feinde und damit sich selbst. Eine politische Begrifflichkeit entwickelt sich, für die es in Ägypten keine Entsprechung gibt. Begriffe wie Volk, Gemeinschaft, Gemeinde, Bund sowie überhaupt die Gattung der Geschichtsschreibung als Form kollektiver Selbstthematisierung oder nationaler Autobiographie sucht man in Ägypten vergeblich.

Die Rolle des providentiellen Feindes findet aber auch in Ägypten eine späte, aber darum nur umso kennzeichnendere Besetzung. Seth hat jetzt die Züge eines asiatischen Eroberers angenommen, der den legitimen ägyptischen König, Horus, vom Thron stößt und seine mörderische Gewalt und gesetzlose Brutalität vor allem an den Tempeln und ihren Göttern auslässt. Er verzehrt die heiligen Tiere, verletzt die Tabus und streicht die Opferlieferungen. Er verkörpert den Inbegriff des Religionsfrevlers. Sein Ziel ist die Zerstörung der Kulte, aber damit geht die ganze Welt zugrunde, denn die Kulte haben die Funktion, die Welt in Gang zu halten. Wenn Seth sein Ziel erreicht, geht nicht nur die ägyptische Kultur zugrunde, sondern die Welt unter.

So wie sich in den Zeiten politischer Unsicherheit die ägyptischen Städte mit Mauern umgeben und in Zitadellen verwandeln, so umgibt sich unter den Bedingungen der Fremdherrschaft die ägyptische Kultur mit symbolischen Mauern und Schutzzonen. Wir müssen auch hierin Reaktionsbildungen auf die historischen Erfahrungen der Perser- und Ptolemäerzeit erblicken. Sie finden ihre genaue Entsprechung in Judäa, wo sich die jüdische Kultur mit der symbolischen Mauer des Gesetzes gegen die persische und hellenistische Bedrohung ihrer kulturellen

$38 \operatorname{Ex} 17,14$.

39 Dtn 25,17-19.

40 Nach der hebräischen Inschrift auf dem Mémorial du martyr juif inconnu in Paris, 17 rue Geoffroyl'Asnier (eigene Übersetzung). 
Identität schützt. Im Brief des Aristeas aus dem 2. Jh. von Chr. wird diese fortifikatorische Metaphorik ausgeführt ${ }^{41}$ :

Der Gesetzgeber, von Gott zu umfassender Erkenntnis ausgerüstet, umschloß uns mit nicht zu durchbrechenden Palisaden und ehernen Mauern, damit wir mit keinem der anderen Völker in irgendeiner Hinsicht in Verkehr seien, rein an Leib und Seele, frei von trügerischen Vorstellungen, den Gott, der allein Gott, allein mächtig ist, im Unterschied zur Schöpfung verehrten [...] Damit wir nun mit nichts uns befleckten und nicht im Verkehr mit Schlechtem verdorben würden, umschloß er uns von allen Seiten mit Reinheitsvorschriften, Geboten über Essen und Trinken, Berühren, Hören und Sehen.

Mary Douglas hat diese Haltung als die typische Einstellung einer „Enklavenkultur" gedeutet, die sich mit einem Wall ritueller Reinheitsgrenzen umgibt, um dem Gleichschaltungsdruck einer feindlichen Umwelt zu widerstehen. ${ }^{42}$ In Ägypten haben wir es mit einem vergleichbaren Prozess symbolischer Befestigung zu tun. Die symbolischen Grenzen heißen hier bwt „Abscheu, Tabu“ (Reinheit/Unreinheit) und šst' „Geheimnis“ (Wissen/Verrat). Hierher gehören vor allem die phantastisch anmutenden, aber wahrscheinlich nicht aus der Luft gegriffenen Angaben Herodots über die strikten Reinheitsvorschriften, die die Ägypter im Verkehr mit Griechen und vermutlich Ausländern überhaupt beachteten. Kein Ägypter würde ein Messer oder irgendein Kochgerät berühren, welches zuvor ein Grieche benutzt habe und kein Fleisch von einem Tier essen, das mit einem griechischen Messer geschlachtet worden sei. Ferner würde kein Ägypter es über sich bringen, einen Griechen auf den Mund zu küssen. ${ }^{43}$ Sicher haben beide Kategorien der Absonderung und der Markierung von Differenz - Abscheu und Geheimnis, Verunreinigung und Verrat - in Ägypten eine lange Tradition. Sie haben ihre traditionelle Funktion aber in der Differenz zwischen dem Heiligen und dem Profanen, und nicht zwischen dem Eigenen und dem Fremden. Die Tabuvorschriften gelten speziell dem Priester, nicht dem Ägypter allgemein. Er hat sich für den Umgang mit dem Heiligen strengen Reinheits- und Enthaltsamkeitsvorschriften zu unterwerfen, durch deren Befolgung er sich von der profanen Menge abgrenzt. Dasselbe gilt für die Kategorie des Geheimnisses, die Arcandisziplin, der die Priester unterworfen werden. Sie soll die heiligen Riten vor Profanation schützen. Dabei ist nicht an Ausländer, sondern an Leute gedacht, die nicht eingeweiht sind.

Unsere Betrachtung der Sentenz „Der für die Wahrheit Taube hat keinen Freund" hat uns weit vom Thema „Freundschaft" weggeführt. Daher ist es notwendig, abschließend noch einmal auf diesen Ausgangspunkt zu verweisen. Der Satz ist pars pro toto zu verstehen, als konkrete Exemplifizierung des Prinzips, das nach ägyptischer Vorstellung die Welt im Innersten zusammenhält. Das ist die

\footnotetext{
41 Brief des Aristeas 139 und 142, in: Gerhard Delling, Die Bewältigung der Diasporasituation durch das bellemistische Judentum, Berlin 1987, S. 9.

42 Mary Douglas, In the Wilderness, Sheffield 1993.

43 Herodotos, hrsg. von Heinrich Stein, Bd. II, Berlin 1856, ND 1968, S. 41.
} 
Ma'at - Wahrheit - Gerechtigkeit - Ordnung - Harmonie -, die die gesamte menschliche und kosmische Welt durchwaltet und an der der Einzelne nur Anteil gewinnt, wenn er zuhören kann.

\section{Bibliographie}

Das Ägytische Totenbuch, übers. u. komm. von Gregoire Kolpaktchy, Frankfurt a. M. 1970. Aristoteles: Nikomachische Ethik, übers. u. komm. von Franz Dirlmeier, Berlin 1979.

Assmann, Aleida: Der lange Schatten der Vergangenheit. Erinnerungskultur und Geschichtspolitik, München 2006.

Assmann, Jan: Der König als Sonnenpriester. Ein kosmographischer Begleittext zur kultischen Sonnenbymnik, in thebanischen Tempeln und Gräbern, Stuttgart u. a. 1970.

- Sonnenbymnen in Thebanischen Gräbern, Mainz 1983.

- „Der schöne Tag. Sinnlichkeit und Vergänglichkeit im altägyptischen Fest", in: Das Fest, hrsg. von Walter Haug, Rainer Warning, München 1989.

- Ma'at. Gerechtigkeit und Unsterblichkeit im Alten Ägypten, München 1990.

- Stein und Zeit. Mensch und Gesellschaft im Alten Ägypten, München 1991.

- „Zur Geschichte des Herzens im alten Ägypten“, in: Die Erfindung des Inneren Menschen. Studien zur religiösen Anthropologie, hrsg. von Jan Assmann, Theo Sundermeier, Gütersloh 1993 , S. 81-112.

- Ägypten. Eine Sinngeschichte, Frankfurt a. M. 2000.

Die autonome Person - eine europäische Erfindung?, hrsg. von Klaus Peter Koepping, Michael Welker, Reiner Wiehl, München 2002.

Die Bibel. Altes und Neues Testament. Einbeitsübersetzung, Freiburg u. a. 1980.

Brunner, Hellmut: „Das Herz im ägyptischen Glauben“, in: ders.: Das Herz im Umkereis des Glaubens, Biberach 1965, S. 81-106.

Däubler, Theodor: Hymne an Italien, Leipzig 1919.

Delling, Gerhard: Die Bewältigung der Diasporasituation durch das bellenistische Judentum, Berlin 1987.

Derrida, Jacques: Gesetzeskeraft. Der mystische Grund der Autorität, Frankfurt a. M. 1991.

Douglas, Mary: In the Wilderness, Sheffield 1993.

Herodotos, hrsg. v. Heinrich Stein, Bd. II, Berlin 1856, ND 1968.

Hornung, Erik: Altägyptische Dichtung, Stuttgart 1996.

Ma'at Konfuzius Goethe. Drei Lebren für das richtige Leben, hrsg. von Jan Assmann, Ekkehard Krippendorff, Helwig Schmidt-Glitzer, Frankfurt a. M. 2006.

Meier, Heinrich: Carl Schmitt, Leo Strauss und der „Begriff des Politischen“. Zu einem Dialog unter Abwesenden, Stuttgart 1998.

- Die Lehre Carl Schmitts. Vier Kapitel zur Unterscheidung politischer Theologie und politischer Philosophie, Stuttgart 1994.

Klasens, Adolf: A Magical Statue Base (Socle Behague) in the Museum of Antiquities at Leiden, Leiden 1952.

Krippendorf, Ekkehard: „Freundschaft als politische Kategorie“, in: ders.: Die Kultur des Politischen. Wege aus den Diskursen der Macht, Berlin 2009, S. 45-54.

Pascal, Blaise: Pensées et opuscules, hrsg. von Léon Brunschvicg, Paris 1912. 
Sander-Hansen, Constantin-Emil: Die Texte der Metternichstele, Kopenhagen 1956.

Schmitt, Carl: Der Begriff des Politischen. Text von 1932 mit einem Vorwort und drei Corollarien, unveränderter ND der 1963 erschienenen Auflage, Berlin 1979.

Sternberg, Heike: „Die Metternichstele“, in: Texte aus der Umwelt des Alten Testaments (TUAT), hrsg. von Otto Kaiser, Bd. II.3 (Rituale und Beschwörungen II), Gütersloh 1988, S. 376.

Urkunden mythologischen Inhalts VI, hrsg. von Siegfried Schott, Leipzig 1929-1939. 